\title{
$4 \quad$ Ethical principles and our responsibilities
}

As more digital tools are deployed in health research and care settings, new questions emerge about how to use them responsibly and ethically. Anyone developing and/or testing a digital tool for use in disease prevention and treatment within the USA should be aware of the regulatory requirement to obtain institutional review board (IRB) approval prospectively when involving people as research participants.

\section{History}

The development of the IRB peer review process stemmed from egregious acts whereby researchers disregarded the rights and welfare of research participants. One example, known as the 'Tuskegee Study of Untreated Syphilis in the Negro Male', was an observational study of the natural progression of syphilis initiated by the Public Health Services in 1932.1,2 At that time, there was no treatment for syphilis; however, after penicillin was developed, the study participants were not treated and the study continued for nearly 40 years. The National Research Act was passed in 1974; it involved creating a National Commission for the Protection of Human Subjects of Biomedical and Behavioral Research with a goal of preventing future atrocities. It was this Commission that required the formation of IRBs and also wrote the Belmont Report. Published in 1979, the Belmont Report describes three guiding principles of ethical biomedical and behavioral research: respect for persons, beneficence and justice.

As cybersecurity concerns increased with research involving information and communication technologies (ICT), the Department of Homeland Security developed the Menlo Report. Published in 2012, this Report adapted the ethical principles of the Belmont Report to ICT research and added a fourth principle - that of respect for the law and public interest, which focuses on transparence of potential conflicting interests and accountability of stakeholders. ${ }^{3}$

Informed by guiding ethical principles, a federal policy for the protection of human subjects was published in 1991 and adopted 
by 15 federal agencies and institutes. ${ }^{4}$ The regulations speak to basic protections for human research participants in subpart A, known as the Common Rule. Additional subparts speak to protections for vulnerable populations, including children and prisoners.

\section{Core principles}

Here, we describe the ethical principles and how the regulations and Common Rule are implemented in practice. The three core principles of biomedical ethics described in the Belmont Report are at the core of research ethics and should be carefully considered during the study design phase and ethics review process. ${ }^{5}$

Respect for persons is demonstrated through the informed consent process, which occurs when a person is given the information needed to make a sound decision about whether to volunteer to willingly participate. How this information is conveyed is important because volunteering to be a research participant is different from, say, accepting terms of service (ToS) to access an app, or signing a consent form to obtain medical care. In the latter, a person will not be able to access the app if they do not accept the ToS nor will they receive medical care if they do not sign the medical consent form. Consent to participate in research is a choice that an individual can only make if presented with information in a setting conducive to good decisionmaking. There can be no coercive actions (e.g. high incentive payments, free services) that may compromise an individual's ability to volunteer. The informed consent process involves more than signing a form to document voluntary participation - it is the first of what may be many interactions between a participant and the research team and is part of developing a trusted relationship.

Technological literacy is another important consideration. For informed consent to be meaningful, participants will need to be 'tech-literate' enough to understand the specifics of how their data will be obtained and used. Likewise, concerns about privacy are often raised when discussing the passive and ubiquitous nature of the tools used in digital medicine. ${ }^{6}$ Attitudes and preferences also vary across generations, with older adults preferring more privacy control compared with teens and young adults. ${ }^{7}$ 
Education. All these concerns suggest a need to better educate prospective participants - and yet, integrating these concepts into the consent process is not easy. Moving forward, this charge will require a commitment from the medical community to provide accessible public-facing educational modules. For example, one way to improve tech literacy might be to include a brief animation describing the difference between de-identification and anonymity when describing data-sharing practices, or an illustration of what it means to store data in a cloud. A participant may also think that if the study team has access to their health data in real time, $24 / 7$, then that means someone is paying attention to them (which may not be the case). Clarifying these concepts is important and how best to do this will require experts in instructional design who can deliver creative educational content. One organization working to advance meaningful informed consent in digital health research is Sage Bionetworks; it has published a toolkit to assist researchers. ${ }^{8}$

Beneficence is where the probability and magnitude of potential harms are weighed against the possible benefits to a participant, the people they represent and society. Determining risk of harm is a somewhat subjective process, yet worth breaking down. We need to consider potential sources of harm and try to quantify the likelihood of something going wrong as well as the consequences. For instance, if a technology collects and then transmits a study participant's location data to a publicly accessible or non-secure website, the likelihood of a loss of privacy is $100 \%$ for all users - yet the consequences will vary. For most people, these will be negligible, but for a domestic abuse survivor or undocumented migrant, the consequences might be severe. Thus, the same potential harm presents a low risk for most, but a high risk for some important groups.

Safeguarding data and managing data-sharing protocols are important considerations when applying the principle of beneficence, and researchers, IRBs and research participants need to think about carefully these. When using third-party commercial apps or measurement tools, it is critical that ToS and end user license agreements (EULAs) be reviewed to ensure they do not introduce unnecessary risks to the end user, be it a research participant or patient. 
Harm. Other factors specific to risk assessment include the type of potential harm (e.g. physical, psychological, economic, social) as well as the duration and severity of harm to research participants. Research is inherently risky because we are learning something that is not yet known. Research participants are often told about risks as an odds ratio. For example, in studies that include a test for maximum oxygen uptake, participants are required to exercise to exhaustion. There is a 1 in 12000 chance that a healthy individual doing this study will have a cardiac event that may lead to death. Because of this particular risk of harm, the research team can mitigate risk by having access to personnel and equipment used to treat a cardiac event. Having this information, an individual can decide whether they want to take that chance or not.

Validity and reliability. Within the domain of beneficence is the need for the digital measurement tools to be valid and reliable (see chapter 9). There is no potential benefit of knowledge gain if the study is poorly designed and the tools are not trustworthy. The old adage 'garbage in, garbage out' is a serious concern and one that must be addressed by doing the appropriate studies early to ensure the products, regardless of whether there is a medical claim, are safe and produce useful data.

Justice. The principle of justice focuses on the fair distribution of the benefits and burdens of research and recruitment protocols that are inclusive of those most likely to benefit from knowledge gained. With digital tools, we have the opportunity to reach a more diverse audience, including those in communities where health disparities are most prevalent. To do that requires that we design technologies that are accessible and, in some cases, culturally tailored. With that in mind, including end users in the development process who represent a wide cross-section of our society is one way we can be responsive to the principle of justice.

For example, in a study designed to increase physical activity in refugee women, the researcher decided to use a wrist-worn accelerometer to assess daily movement. The participants were given the sensor and shown how to use it. One week later, the researcher returned to gather the measurement tools and found that no data had been collected. Turns out a wrist-worn mobile technology was 
culturally unacceptable and drew unwanted attention to the women, so they did not wear it. ${ }^{9}$ This story sheds light on the fact that while digital tools should improve access to health research and healthcare, they can also perpetuate disparities and prevent access if not well designed and deployed.

\section{Key points - ethical principles and our responsibilities}

- The principles that guide the ethical conduct of biomedical and behavioral research include: respect for persons, beneficence, justice (Belmont Report) and respect for law and public interest (Menlo Report).

- Knowing how to apply ethical principles is a responsibility of all stakeholders involved in digital health research, including technologists, researchers and ethics review boards.

\section{References}

1. Centers for Disease Control and Prevention. US Public Health Service Syphilis Study at Tuskegee.

The Tuskegee Timeline, 2016. www.cdc.gov/tuskegee/timeline.htm, last accessed 25 August 2019.

2. Chadwick GL. Historical perspective: Nuremberg, Tuskegee, and the radiation experiments. J Int Assoc Physicians AIDS Care 1997;3:27-8.

3. US Department of Homeland Security. The Menlo Report: Ethical Principles Guiding Information and Communication Technology Research, 2012. www.dhs.gov/sites/default/ files/publications/ CSDMenloPrinciplesCORE20120803_1.pdf, last accessed 24 September 2019.
4. Office for Human Research Protections. Federal Policy for the Protection of Human Subjects ('Common Rule'), 2016. www.hhs.gov/ohrp/regulations-andpolicy/regulations/common-rule/ index.html, last accessed 24 September 2019.

5. The National Commission for the Protection of Human Subjects of Biomedical and Behavioral Research, Department of Health, Education and Welfare (DHEW). The Belmont Report. Washington: US Government Printing Office, 1978. https:// videocast.nih.gov/pdf/ohrp belmont_report.pdf, last accessed 31 July 2019. 
6. Filkins BL, Kim JY, Roberts B et al. Privacy and security in the era of digital health: what should translational researchers know and do about it? Am J Transl Res 2016;8:1560-80.

7. Wang S, Bolling K, Mao W et al. Technology to support aging in place: the older adult perspective. Healthcare 2019;7:E60.
8. Sage Bionetworks. Elements of Informed Consent, 2019. https:// sagebionetworks.org/tools_resources/ elements-of-informed-consent, last accessed 25 August 2019.

9. Nebeker C, Murray K, Holub C et al. Acceptance of mobile health in communities underrepresented in biomedical research: barriers and ethical considerations for scientists. JMIR Mhealth Uhealth 2017;5:e87. 